\title{
PTEN Inhibition to Facilitate Intrinsic Regenerative Outgrowth of Adult Peripheral Axons
}

\author{
Kimberly J. Christie, ${ }^{1,2}$ Christine A. Webber, ${ }^{1,2}$ Jose A. Martinez, ${ }^{1,2}$ Bhagat Singh, ${ }^{1,2}$ and Douglas W. Zochodne ${ }^{1,2}$ \\ ${ }^{1}$ Department of Clinical Neurosciences and ${ }^{2}$ Hotchkiss Brain Institute, University of Calgary, Calgary, Alberta T2N 4N1, Canada
}

In vivo regeneration of peripheral neurons is constrained and rarely complete, and unfortunately patients with major nerve trunk transections experience only limited recovery. Intracellular inhibition of neuronal growth signals may be among these constraints. In this work, we investigated the role of PTEN (phosphatase and tensin homolog deleted on chromosome 10) during regeneration of peripheral neurons in adult Sprague Dawley rats. PTEN inhibits phosphoinositide 3-kinase (PI3-K)/Akt signaling, a common and central outgrowth and survival pathway downstream of neuronal growth factors. While PI3-K and Akt outgrowth signals were expressed and activated within adult peripheral neurons during regeneration, PTEN was similarly expressed and poised to inhibit their support. PTEN was expressed in neuron perikaryal cytoplasm, nuclei, regenerating axons, and Schwann cells. Adult sensory neurons in vitro responded to both graded pharmacological inhibition of PTEN and its mRNA knockdown using siRNA. Both approaches were associated with robust rises in the plasticity of neurite outgrowth that were independent of the mTOR (mammalian target of rapamycin) pathway. Importantly, this accelerated outgrowth was in addition to the increased outgrowth generated in neurons that had undergone a preconditioning lesion. Moreover, following severe nerve transection injuries, local pharmacological inhibition of PTEN or siRNA knockdown of PTEN at the injury site accelerated axon outgrowth in vivo. The findings indicated a remarkable impact on peripheral neuron plasticity through PTEN inhibition, even within a complex regenerative milieu. Overall, these findings identify a novel route to propagate intrinsic regeneration pathways within axons to benefit nerve repair.

\section{Introduction}

Clinical injuries to peripheral nerves are common and recovery is highly limited. Only $10 \%$ of axons may reconnect to targets after a transection injury (Witzel et al., 2005). Many constraints on axon regrowth are extrinsic, such as limited growth factor support by glial cells, macrophages, or mast cells. Inhibitory molecules in the matrix may activate RHOA GTPases to induce growth cone collapse (Niederöst et al., 2002; Jain et al., 2004), or the outgrowth zone may be ischemic, exposed to nitrative stress, or physically distorted to inhibit growth (Lyons and Woodhall, 1949).

Amplification of the PI3K/Akt (protein kinase B) pathway leads to increased axon growth, originally shown in PC12 cells (Soltoff et al., 1992; Korhonen et al., 1999). Key members of this

\footnotetext{
Received Dec. 18, 2009; revised May 11, 2010; accepted May 18, 2010.

The work was supported by an operating grant from the Canadian Institutes of Health Research. K.J.C. was supported by a Graduate Studentship of the Alberta Heritage Foundation for Medical Research (AHFMR) and the National Science and Engineering Research Council. C.A.W. was a Postdoctoral Fellow of AHFMR and D.W.Z. was a Scientist of AHFMR. K.J.C. completed much of the experimental work on this project, contributed to the experimental design and conception, compiled the results, and cowrote the manuscript. C.A.W. contributed to the experimental work, experimental design, and conception of the work and assisted in manuscript writing. J.A.M. and B.S. contributed to experimental work and design of the projects. D.W.Z. supervised the project, contributed to the experimental design, conception, and interpretation of the results, and cowrote the manuscript. We acknowledge the input of GuiFang Guo for technical support.

The authors declare no competing financial interests.

Correspondence should be addressed to Dr. Douglas W. Zochodne, Department of Clinical Neurosciences and the Hotchkiss Brain Institute, University of Calgary, 168 HMRB, 3330 Hospital Drive NW, Calgary, AB T2N 4N1, Canada. E-mail: dzochodn@ucalgary.ca.

DOI:10.1523/JNEUROSCI.6271-09.2010

Copyright $\odot 2010$ the authors $\quad 0270-6474 / 10 / 309306-10 \$ 15.00 / 0$
}

pathway are important for the regulation of survival and growth in neurons, including Akt, GSK3 $\beta$ (glycogen synthase kinase $3 \beta$ ), and PTEN. Specifically, phosphorylation of Akt and subsequent inhibition of GSK $3 \beta$ are closely linked to axon outgrowth (Eickholt et al., 2002; Chin et al., 2005). PTEN is an endogenous inhibitor of the PI3K pathway and recently has been shown to be important for central axon growth (Park et al., 2008). By acting as the phosphatase that converts $\operatorname{PIP}(3)$ (phosphatidylinositol 3,4,5 triphosphate) to $\mathrm{PIP}(2)$ (phosphatidylinositol 4,5 diphosphate), PTEN acts to attenuate PIP(3) activation of Akt. One action of PTEN in the unphosphorylated state and at the lipid membranes is to dampen regenerative activity in conjunction with other inhibitory molecules, such as Semaphorin 3A (Sema3A) and GSK3 $\beta$ (Chadborn et al., 2006). Recent work has also suggested that PTEN attenuates regrowth of injured CNS axons by suppression of mTOR (Park et al., 2008).

There have been different approaches used to activate the PI3K pathway in neurons. One approach involves exogenous application of growth factors, such as nerve growth factor (NGF) (Jones et al., 2003; Kemp et al., 2007). Another uses knock-out of PTEN; however, the loss of PTEN is oncogenic and a long-term knock-out in particular tissues might lead to neoplasia (Ali et al., 1999). Therefore, our approach to amplifying this pathway was to temporarily reduce the endogenous inhibitor of the pathway, PTEN, specifically within the injury milieu of the regenerating peripheral nerve trunk. This novel approach has not been used to study regeneration in peripheral neurons.

In this work, we addressed PTEN expression in peripheral neurons and axons and whether it constrains the intrinsic regen- 
A
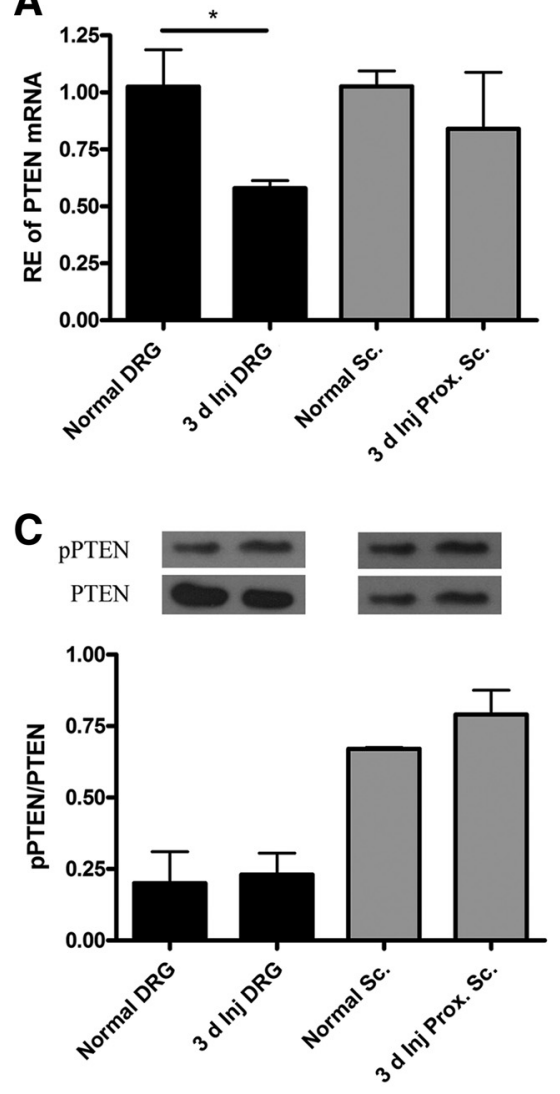
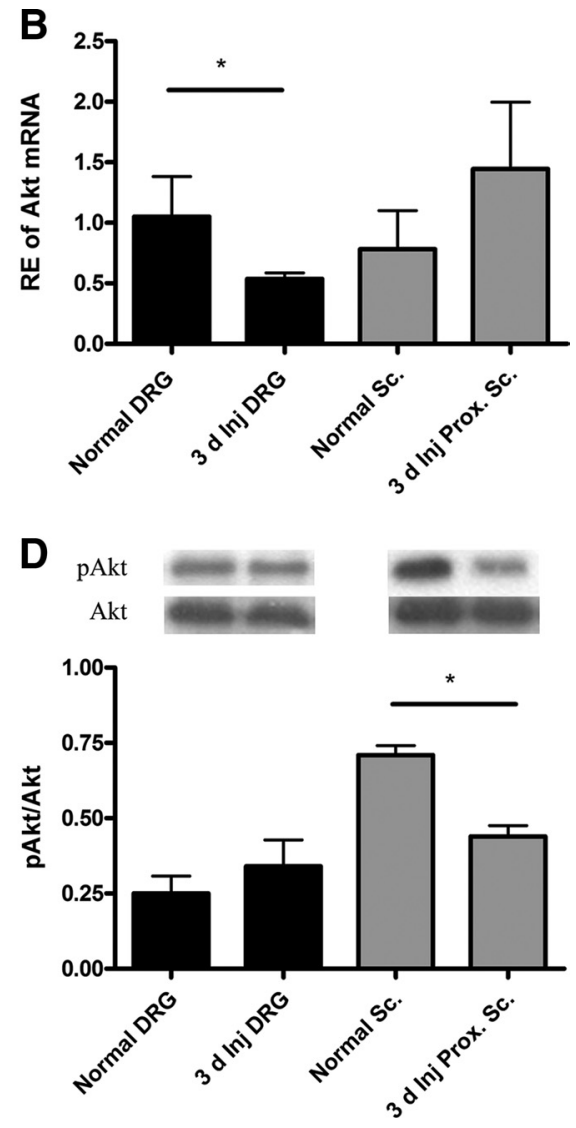

Figure 1. PTEN and Akt mRNA and protein are present in the adult DRG and sciatic nerve. $A, q R T-P C R$ for PTEN in normal and $3 \mathrm{~d}$ injured DRG and sciatic nerve. $\boldsymbol{B}$, qRT-PCR for Akt in normal and $3 \mathrm{~d}$ injured DRG and sciatic nerve. $\boldsymbol{C}$, Western blot analysis of pPTEN/total PTEN in normal and $3 \mathrm{~d}$ injured DRG and sciatic nerve. $\boldsymbol{D}$, Western blot analysis of pAkt/total Akt in normal and $3 \mathrm{~d}$ injured DRG and sciatic nerve. Asterisks indicate significant differences ( $t$ test, $p<0.05, n=3-6$ ). Error bars represent SEM.

erative capacity of peripheral neurons both in vitro and in vivo. We used a peripheral nerve transection injury, a severe yet common nerve lesion that offers a "worst case" scenario to test novel forms of regenerative support. We then manipulated PTEN mRNA and protein both in vitro and in vivo to determine its role in peripheral nerve regeneration. The results indicated that this phosphatase brake is widely expressed in peripheral neurons, including those that have been injured. Importantly, we found inhibition of PTEN augments axonal outgrowth, even beyond that expected of preconditioning, and unlike the CNS, this impact appears independently of the mTOR pathway.

\section{Materials and Methods}

Animals and preconditioning lesion experiments. Adult male Sprague Dawley rats (Charles River Laboratories) with an initial weight of 200 $300 \mathrm{~g}$ were housed in plastic cages with a normal light/dark cycle and ad libitum access to rat chow and water. All protocols were reviewed and approved by the University of Calgary Animal Care Committee using the Canadian Council of Animal Care guidelines. Experiments were designed to minimize the number of animals required and to minimize animal suffering as per the Canadian Council on Animal Care. Rats were anesthetized with isoflurane (Abbot Laboratories), and an injection of $0.25 \mathrm{ml}$ of buprenorphine was given for analgesia. For preconditioning, the sciatic nerve was cut at the midthigh level under aseptic conditions. The skin wound was sutured with 3-0 silk suture, and the rats were caged individually for 3 or $7 \mathrm{~d}$. Buprenorphine was given on days 1 and 2 following the injury.

Quantitative reverse transcription PCR. Total RNA was extracted from tissues using Trizol reagent as per the manufacturer's instructions (In- vitrogen). One microgram of total RNA was treated with DNase (Promega) and processed to cDNA synthesis using the SuperScript II Reverse Transcriptase (Invitrogen). All PCR primers were designed using software Primer Express 2.0 (Applied Biosystems).

Primer sequences were as follows: $18 \mathrm{~S}$ F, $5^{\prime}$ TCCCTAGTGATCCCCGAGAAGT-3'; 18S R, 5'-CCCTTAATGGCAGTGATAGCGA-3'; AKT R, 5'-TTTGTCATGGAGTACGCCAATG-3'; AKT F, 5' -CACAATCTCCGCACCGTAGAA3'; PTEN F, 5'-GACGACAATCATGTTGCAGCA-3'; PTEN R, 5' -GCCTTTAAAAACTTGCCCCG-3'.

Akt, PTEN, or $18 \mathrm{~S}$ products were labeled using SybrGreen (Invitrogen). All reactions were performed in duplicate in an ABI PRISM 7000 Sequence Detection System (Applied Biosystems) and analyzed using the $2 \Delta \Delta$ cycle threshold method, and results are presented as the fold induction of mRNA for Akt and PTEN in injured L4-L5 dorsal root ganglion (DRG) neurons or sciatic nerve normalized to $18 \mathrm{~S}$, compared to normal, uninjured L4-L5 DRG neurons or sciatic nerve (defined as 1.0 -fold). Statistical analysis was performed using oneway ANOVA with Tukey post hoc analysis.

Western immunoblots. Before protein extraction, DRGs were put into single-cell suspension and partially purified. Approximately 1 $\mathrm{cm}$ of uninjured and $0.5 \mathrm{~cm}$ of $3 \mathrm{~d}$ injured proximal and distal segments of sciatic nerve were fresh frozen in liquid nitrogen. Both DRG and sciatic nerves were placed into a lysis/RIPA buffer [contents $50 \mathrm{~mm}$ HEPES (SigmaAldrich), pH 7.4, $150 \mathrm{~mm} \mathrm{NaCl}$ (EM Science), $10 \%$ glycerol (VWR International), $1.5 \mathrm{~mm}$ $\mathrm{MgCl}_{2}$ (Sigma-Aldrich), 1 mm EGTA (SigmaAldrich), $1 \mathrm{~mm}$ sodium vanadate (SigmaAldrich), $10 \mathrm{~mm}$ sodium pyrophosphate (Sigma-Aldrich), 10 mm NaF (Sigma-Aldrich), 1\% Triton X-100 (SigmaAldrich), 1\% sodium deoxycholate (Sigma-Aldrich), 0.1\% SDS (BioRad), $1 \mathrm{~mm}$ PMSF (Sigma-Aldrich), and $1 \times$ Complete mini Protease Inhibitor (Roche Diagnostics)]. Sciatic nerves were homogenized with glass sliders on ice (Wheaton) and then centrifuged at $10,000 \times g$ for 10 $\mathrm{min}$. The supernatant was stored at $-20^{\circ} \mathrm{C}$ before SDS-PAGE and immunoblotting analysis. Protein concentration determined by a BCA Protein Assay Kit (Pierce). Equal amounts $(50 \mu \mathrm{g})$ of protein were loaded in each well and samples were separated by SDS-PAGE using $8 \%$ polyacrylamide [resolving gel: $30 \%$ polyacrylamide mixture (Bio-Rad) with $1.5 \mathrm{M}$ Tris, pH 8.8 (Sigma-Aldrich), 10\% SDS, 10\% ammonium persulfate (Invitrogen), and $0.06 \%$ Ultrapure Temed (Invitrogen); stacking gel: $30 \%$ acrylamide, $1.0 \mathrm{~m}$ Tris, $\mathrm{pH} 6.8,10 \%$ SDS, $10 \%$ ammonium persulfate, and $0.1 \%$ Temed]. Gels were placed in a running buffer [30 mM Tris, 144 mM glycine (Fisher Scientific), and $0.1 \% \mathrm{v} / \mathrm{v}$ SDS] at $90 \mathrm{~V}$ for $10 \mathrm{~min}$ until the loading dye passed through the stacking gel and then increased to 150 $\mathrm{V}$ until the migrating dye front reached the bottom of the gel. Separated proteins were then transferred onto PVDF membrane (Bio-Rad) for $1.5 \mathrm{~h}$ at $100 \mathrm{~V}$ in transfer buffer [ $30 \mathrm{~mm}$ Tris, $144 \mathrm{~mm}$ glycine, and $20 \% \mathrm{v} / \mathrm{v}$ methanol (Fisher Scientific)]. The immunoblot was blocked for $30 \mathrm{~min}$ in 5\% (w/v) milk (Nestle, Carnation) in TBST [12.1 mM Tris, $81.9 \mathrm{~mm}$ $\mathrm{NaCl}$, and $0.1 \%(\mathrm{v} / \mathrm{v})$ Tween 20 (VWR International)]. Primary antibodies to PTEN (1:25,000, anti-rabbit), phosphorylated PTEN (pPTEN) (Ser 380), Akt, pAkt (Ser 473), GSK-3 $\beta$, and pGSK-3 $\beta$ (Ser 9, 46 kDa) (Cell Signaling Technology) were added with an anti-actin antibody (1:1000, Santa Cruz Biotechnology) as a loading control overnight at $4^{\circ} \mathrm{C}$. After three rinses for 10 min each in TBST, both secondary antibodies, antirabbit IgG HRP and anti-mouse IgG HRP (Santa Cruz Biotechnology), were incubated with the immunoblot at 1:50,000 dilution for $1.5 \mathrm{~h}$ at 
room temperature. Following three TBST rinses, signal detection was performed via exposing of the blot to enhanced chemiluminescent reagents (Lumi-Light Plus; Roche Diagnostics) for $4 \mathrm{~min}$. The blots were subsequently exposed on Hyperfilm (GE Healthcare) to capture the images of the bands. Analysis was performed using Adobe Photoshop to measure the pixel density of the bands; this was divided by the density of the actin band for a loading control. Statistics were performed using $t$ test analysis.

Immunohistochemistry. Harvested L4 and L5 DRGs, and sciatic nerve (normal or proximal and distal to transection) were placed in modified Zamboni's solution overnight at $4^{\circ} \mathrm{C}$. The samples were then rinsed in PBS three times and suspended in PBS-20\% sucrose solution overnight $\left(4^{\circ} \mathrm{C}\right)$. The samples were aligned into holders and frozen in OCT compound (TissueTek Sakura Finetek). Cryostat sections of 12 $\mu \mathrm{m}$ were taken and placed on slides that were frozen at $-80^{\circ} \mathrm{C}$. For staining the slides were thawed at room temperature and rinsed in PBS. The tissue was permeabilized for $1 \mathrm{~h}$ in $5 \%$ goat serum, $1 \% \mathrm{BSA}$, and $0.3 \%$ Triton-X. The slides were probed for PTEN, pPTEN (Ser 380), Akt, or pAkt (Ser 473) (1:100) (Cell Signaling Technology) (Chadborn et al., 2006; Rankin et al., 2008), and double labeled with NF-200 (1:400) (Sigma-Aldrich), substance P (1:500, Abcam), calcitonin gene-related peptide (CGRP, 1:200, Abcam), or unconjugated IB4 lectin (Vector Laboratories) followed by goat anti-IB4 (1:8000, Vector Laboratories) for $48 \mathrm{~h}$ and then rinsed three times in PBS. Secondary antibodies were sheep anti-mouse IgG CY3 conjugate (1:100, Sigma) and Alexa Fluor 488 goat anti-rabbit IgG $(\mathrm{H}+\mathrm{L})$ conjugate $(1$ : 500, Cedarlane). Secondary antibodies were applied for $1 \mathrm{~h}$, then rinsed three times and mounted with Polyaquamount mounting medium (Polysciences). All samples were imaged with black and white imaging using a Zeiss Axioscope with digital camera and Axiovision imaging software (Zeiss Axioskope, Axiovision, and Axiocam, Zeiss Canada). The green and red pseudocolors were added by Adobe Photoshop software.

Adult sensory neuron cultures. Before tissue harvesting, rats were anesthetized with isoflurane (Abbot Laboratories) and then killed. DRG neurons were dissociated and maintained in vitro using a modification from the method of Lindsay (1988). Briefly, the L4-L6 DRGs were removed from the rats and placed into L15 (Invitrogen) medium, where the axon roots and dural tissue were manually removed. The DRGs were rinsed three times in $\mathrm{L} 15$ medium and then transferred to a tube containing $2 \mathrm{ml}$ of $0.1 \%$ collagenase (Invitrogen) $/ \mathrm{L} 15$. Following a $90 \mathrm{~min}$ incubation at $37^{\circ} \mathrm{C}$, the DRGs were placed into single-cell suspension by triturating $10-15$ times every $7 \mathrm{~min}$ through three $1 \mathrm{ml}$ pipette tips and then three $200 \mu$ l pipette tips. The single-cell suspension was spun for $5 \mathrm{~min}$ at 800 $\mathrm{rpm}(\sim 120 \times g)$ at $4-8^{\circ} \mathrm{C}$, and the cell pellet was washed three times in $2 \mathrm{ml}$ of L15. After the final $5 \mathrm{~min} 800 \mathrm{rpm}$ spin, the cells were resuspended in L15 and passed through a $70 \mu \mathrm{m}$ mesh (VWR International), and then placed in $500 \mu \mathrm{l}$ of L15 enriched with 1:100 dilution of N2 supplement (Invitrogen) and 0.1\% BSA (Sigma) and placed into a culture medium of DMEM/F12 (Invitrogen) + 1:100 dilution N2, 0.5-0.8\% BSA, and 0.2 $\mathrm{ng} / \mathrm{ml} \mathrm{NGF}$ (Cedarlane) plus $50 \mathrm{U}$ of penicillin $\cdot \mathrm{ml}^{-1}, 50 \mathrm{U}$ streptomycin $\cdot \mathrm{ml}^{-1}$ (Invitrogen) and plated onto poly-L-lysine- (SigmaAldrich) and $10 \mu \mathrm{g} / \mathrm{ml}$ mouse laminin- (Invitrogen) coated plates. At the time of plating, a $0,10,50$, or $200 \mathrm{~nm}$ concentration of the PTEN phar- macological inhibitor, dipotassium bisperoxo(pyridine-2-carboxyl) oxovanadate $[\mathrm{bpV}(\mathrm{pic})]$ (EMD Chemicals), was added to the culture medium, along with rapamycin (LC Laboratories) at $50 \mathrm{~nm}$ in other experiments. In separate experiments PTEN siRNA (Cat. \#SI01967084, Rn_PTEN_4, targeting the $3^{\prime}$ region), scrambled siRNA, or transfection reagent alone (Qiagen) were added to the medium at $10 \mathrm{~nm}$ along with the HiPerFect transfection reagent according to the HiPerFect handbook (Qiagen). To verify this siRNA, another siRNA experiment was performed using a second different PTEN siRNA (Cat. \# SI03102806, Rn_PTEN_7, targeting the 5' region). The cells were grown for $18 \mathrm{~h}$ and then fixed and processed for immunocytochemistry. Neurite extension, number of primary neurites (defined as processes extending from the soma), length of the longest neurite, number of branches (defined as a branch point in a primary neurite), and cell body area were analyzed and quantified by MetaXpress software and an observer blinded to condition (Molecular Devices). The MetaXpress program localizes cell bodies based on fluorescent intensity above background. Once a cell body is recognized, it measures the length and number of processes extending from the soma. Statistical analysis was performed using one-way ANOVA with Tukey post hoc analysis.

Immunocytochemistry. DRG cultures were fixed for 30 min with $4 \%$ paraformaldehyde, and then rinsed five times for 5 min each with PBS. 

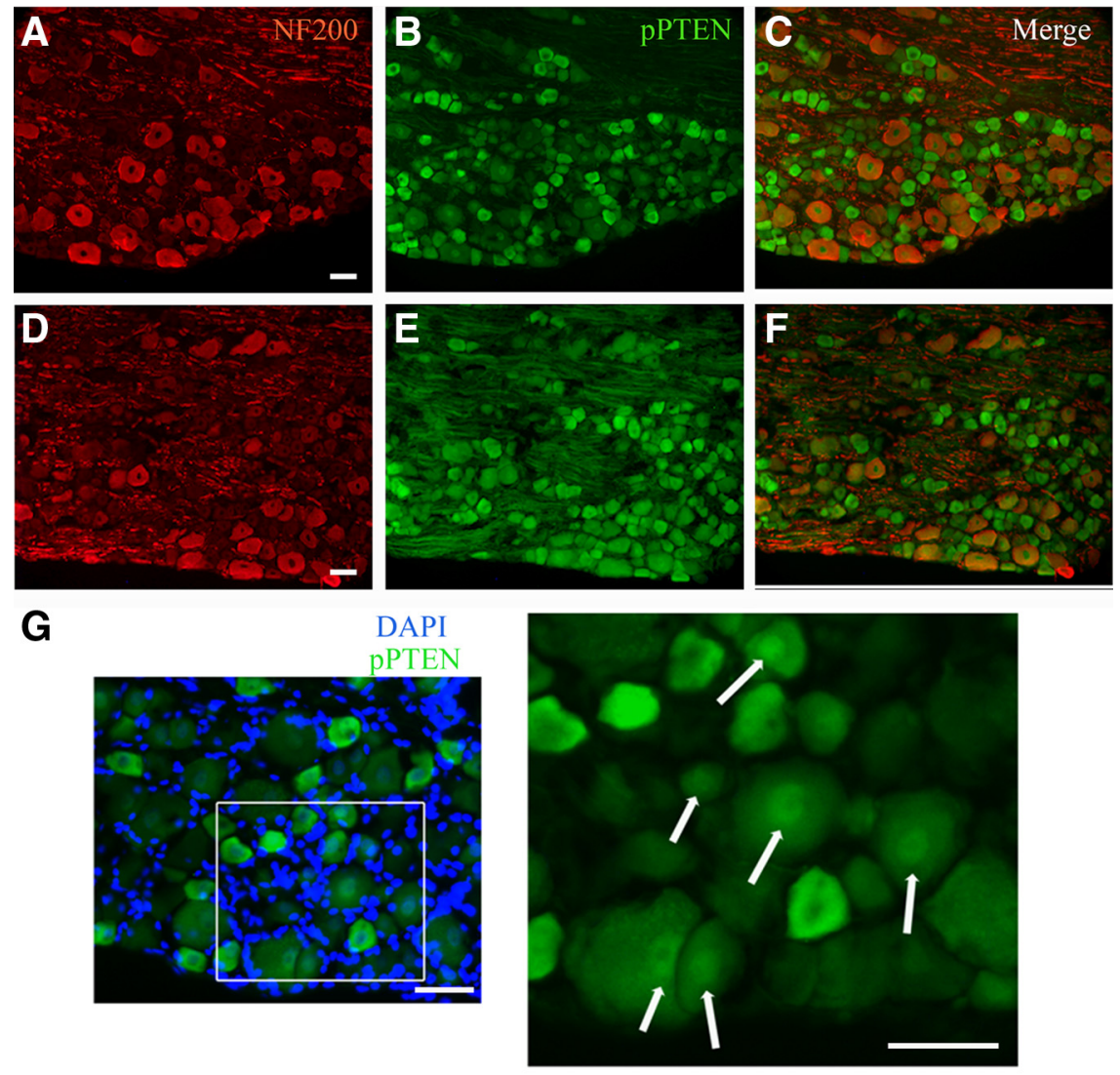

H
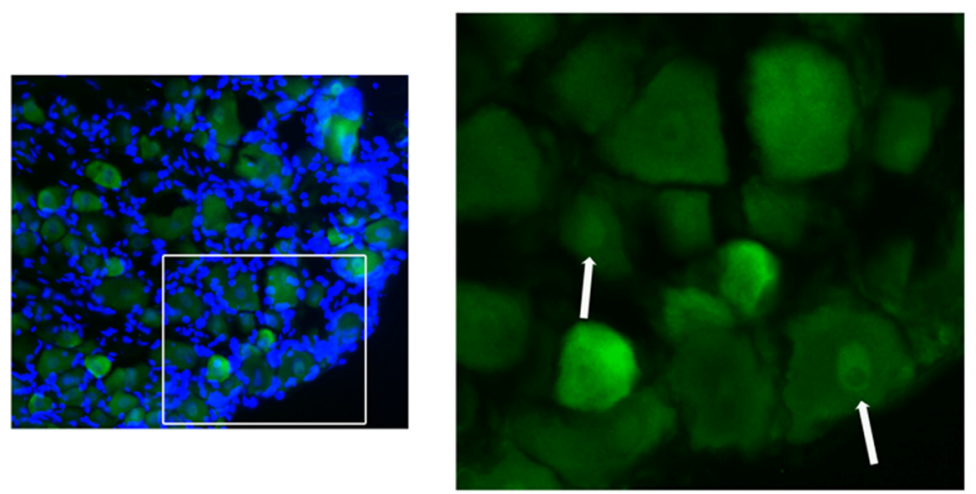

Figure 3. $\quad$ PPTEN expression in DRG nuclei decreases after sciatic nerve injury. $\boldsymbol{A}-\boldsymbol{C}$, Transverse section of normal DRG showing NF200 (A) and pPTEN $(\boldsymbol{B})$ staining and merge $(\boldsymbol{C})$. D $\boldsymbol{F}$, Transverse section of 3 d injured DRG showing NF200 (D) and pPTEN (E) staining and merge $(\boldsymbol{F}) . \boldsymbol{G}, \boldsymbol{H}$, pPTEN staining merged with DAPI in sham $(\boldsymbol{G})$ and injured $(\boldsymbol{H})$ DRG. Panels to the right show magnified nuclear pPTEN expression (arrows). Scale bars are $50 \mu \mathrm{m}$.

The cells were permeabilized for $10 \mathrm{~min}$ in $0.1 \%$ Triton X-100 (SigmaAldrich), then rinsed twice for $5 \mathrm{~min}$ in PBS, and then blocked for $30 \mathrm{~min}$ in PBS with 5\% fetal bovine serum and 0.1\% BSA. PTEN, pPTEN (Ser 380), Akt, pAkt (Ser 473) (1:100), GW182 (gift from Dr. M. Fritzler, University of Calgary, Calgary, Alberta, Canada), or NF200 (1:800) were labeled for $1 \mathrm{~h}$ with a rabbit polyclonal antibodies (Cell Signaling Technology), and this interaction was visualized following 30 min incubation with Alexa Fluor 488 goat anti-rabbit and Cy3 sheep anti-mouse secondary antibodies (Alexis Biochemicals). The cells were plated on to glass slides and immunofluorescence was preserved with Polyaquamount mounting medium (Polysciences). All samples were imaged with black and white imaging using a Zeiss Axioscope with digital camera and Axiovision imaging software (Zeiss Axioskope, Axiovision, and Axiocam, Zeiss Canada). The green and red pseudocolors were added by Adobe Photoshop software.

Regeneration conduit experiments. A $3 \mathrm{~cm}$ incision was made along the length of the lateral aspect of the left thigh, beginning at the sciatic notch and ending near the knee. A second incision was made on the dorsal aspect of the animal superior to the scapulae and parallel to the vertebral column to facilitate insertion of a microinjection port (MIP). Next, blunt forceps were used to tunnel through the subdermal fascia between posterior and anterior incisions. The jaws of the forceps were then used to pull the catheter of the MIP from its implantation site to the incision at the hindlimb. The catheter of the MIP was glued into the access tube of the T-chamber using cyanoacrylate cement. Blunt dissection was performed to expose and mobilize the left sciatic nerve, which was transected at midthigh level using an 11-0 scalpel blade. The proximal and distal stumps of the nerve were then secured into the ends of the nerve chamber using a single 9-0 nylon (Ethicon) suture through the epineurium of each stump, leaving a gap $(3 \mathrm{~mm})$ between stumps. Before closing the wound, a single 4-0 silk suture (Ethicon) was applied to secure the $\mathrm{T}$-intersection of the tube to underlying muscle and second suture was used to reattach the retracted gluteal muscle. Finally, a continuous suture (4-0 silk) is used to close both incisions. At endpoint, nerve chambers were harvested by transection of proximal and distal nerve segments several millimeters from the nerve chamber. Removal of the epineurial sutures freed the regenerate, and the nerve bridge (regenerate) was obtained by lightly pulling the nerve through the chamber. Injections of 0.2 $\mathrm{ml}$ were given on days $0,1,3$, and 5 for PTEN and scrambled siRNA $(2 \mu \mathrm{g} /$ injection $)$ and days $0-6$ for bpv(pic) (200 nM) and saline ( $n=$ 6-8 for each group). Bridges were processed for immunohistochemistry and probed for NF200. At the beginning (first separate highpower field: $400 \times$, each field was $270 \mu \mathrm{m}$ in diameter) of the proximal portion of the regenerative bridge, a clear demarcation could be recognized in the overall structure of the nerve trunk, and the onset of regenerative neurofilament containing axon profiles. Starting at the distal edge of the proximal stump, we counted profiles in a line perpendicular to the direction of the bridge in serial fields distally in high-power fields every $270 \mu \mathrm{m}$ until reaching the last field expressing the heavy chain neurofilament protein, NF200 (neurofilament). For each field, we counted intersecting profiles along the transecting line for axons alone. We counted profiles at each of these sites in three different sections from each rat to determine a mean profile count at each distance from the proximal stump. For each intervention, means and SEs were then calculated for each group of rats ( $n=3$ /group). Counts were done with the observer blinded to the nature of the treatment group (Chen et al., 2005).

\section{Results}

PTEN and downstream mediators of the PI3-K pathway are expressed in the DRG and sciatic nerve both before and following peripheral nerve injury

Little is known about the expression of PTEN in adult peripheral neurons either in vitro or in vivo. Quantitative reverse transcription PCR (qRT-PCR) confirmed the presence of PTEN and Akt mRNA in adult rat sensory neurons of lumbar DRG and sciatic nerve (Fig. $1 A, B$ ). While PTEN and Akt mRNA expression de- 
creased in the DRG after injury, the total amount of phosphorylated protein did not change (Fig. $1 C, D$ ). Within the sciatic nerve, PTEN and Akt mRNA were also expressed (Fig. 1 $A, B$ ); however, immunoblots showed a decrease in phosphorylated Akt (pAkt) at the injury site, where regenerating growth cones reside (Fig. $1 D)(p<$ $0.05, n=3$ ). As PTEN has been shown to be an inhibitor of the PI3K pathway, injured neurons might be expected to downregulate PTEN to facilitate regenerative outgrowth. In support of this, PTEN mRNA levels declined in the DRG at $3 \mathrm{~d}$ following injury (Fig. 1A). However, the overall level of PTEN protein was unchanged at this time point, while pAkt was decreased at the injury site.

Immunohistochemistry for PTEN in lumbar DRGs indicated its presence within neurons, especially in smaller neurofilament-poor neurons (Fig. 2A$C)$. To determine the profile of small neurons that had intense PTEN expression, we stained for CGRP, substance P, and IB4 (binding the lectin Griffonia simplicifolia IB4). Small intensely labeled PTEN neurons did not colocalize with CGRP (Fig. 2D-F) or substance P (data not shown). These neurons did, however, had substantial colocalization with IB4 (Fig. 2G-I). PTEN was expressed in axons (Fig. 2J-L) and Schwann cells (SCs, data not shown) within normal sciatic nerve. Following an injury, there were maintained levels of PTEN in regenerating axons (Fig. $2 M-O$, arrows) and associated SCs (Fig. $2 M-O$, arrowheads) in the proximal stump of the transected sciatic nerve.

pPTEN had similar expression to PTEN in the DRG (Fig. 3A-F). Interestingly, pPTEN was highly expressed in the nuclei of large and medium-sized neurons before injury (Fig. 3G, arrows). Following a sciatic nerve transection, the expression of pPTEN within these nuclei significantly decreased from $57 \pm 14 \%$ (SEM) to $21 \pm 3 \%$ (SEM) (Fig. $3 H)(t$ test, $p<0.05, n=4)$. This finding suggested greater relative cytoplasmic localization and activity.

Two major downstream targets of PI3-K signaling, Akt and GSK $3 \beta$, which have been shown to be influenced by PTEN (Chadborn et al., 2006), were also identified in sensory neurons and axons (Fig. 4). Akt was expressed in axons in the normal sciatic nerve (Fig. $4 G-I$ ) and in the regenerating axons following injury (Fig. $4 J-L$ ). GSK3 $\beta$ showed trends toward lower levels of mRNA after injury in sensory neuron perikarya, with little change in total and inactive phosphorylated protein levels (data not shown). At the injury site, there was a decrease in phosphorylated GSK3 $\beta$ (pGSK3 $\beta$ ) (Fig. 4M) (ratio of pGSK3 $\beta$ /total GSK3 $\beta$ /actin pixel density in normal nerve: $0.77 \pm$ 0.04 to after injury: $0.5 \pm 0.1 \mathrm{SEM}, p<0.05, n=4)$, indicating increased levels of active GSK3 $\beta$, possibly due to a decrease in pAkt (Fig. 1D). Since pAkt phosphorylates and inactivates GSK3 $\beta$, the
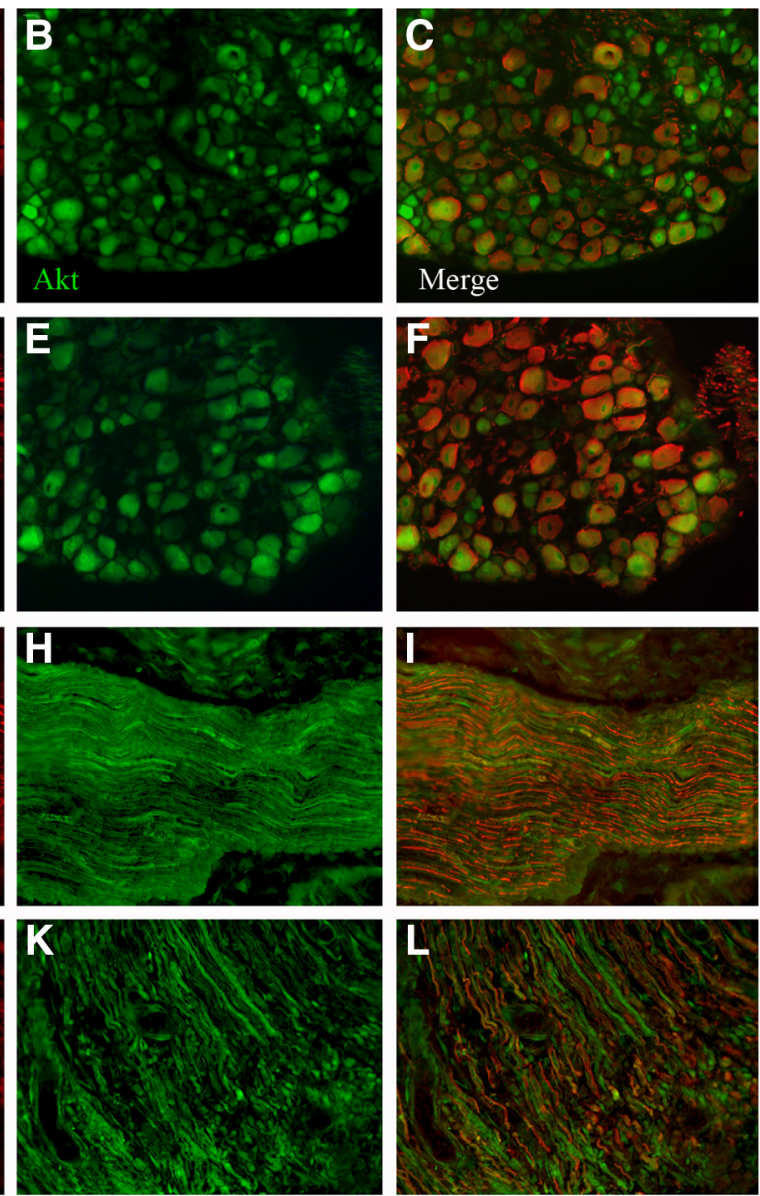

M

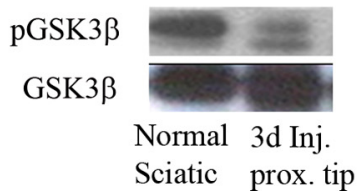

Sciatic prox. tip

Figure 4. Akt is present in the DRG and sciatic nerve and pGSK3 $\beta$ is decreased at the injury site. $\boldsymbol{A}-\boldsymbol{C}$, Transverse section of and 3 d injured $(\boldsymbol{J}-\boldsymbol{L})$ sciatic nerve. $\boldsymbol{M}$, Western blot analysis of pGSK3 $\beta$ (Ser $9,46 \mathrm{kDa}) / G S K 3 \beta(52 \mathrm{kDa})$ in 3 dinjured sciatic nerve. P, Proximal; D, distal; white asterisk indicates transection site.

overall expression results suggested a decline in PI3-K downstream mediators. Together these findings indicated trends in the local changes of pAkt and pGSK $\beta$ that were less supportive for regenerative growth.

Overall, the findings indicated that although PTEN mRNA decreased after injury in the perikarya of sensory neurons, its activated protein (nonphosphorylated), PTEN, was nonetheless expressed and available to attenuate PI3K/Akt signaling, especially at the injury site.

\section{Inhibition of PTEN at the protein level in vitro increases neurite outgrowth}

The ongoing expression of PTEN within injured adult neurons and SCs indicated that it might act as an intrinsic brake on regenerative outgrowth from nerve trunk lesions. Plasticity in both outgrowing axons and partnering SC processes is essential to regenerative growth in peripheral nerves (Chen et al., 2005; McDonald et al., 2006). PTEN was expressed in both sham-injured and injured cultured sensory neurons and glial cells (Fig. 5A-F; 

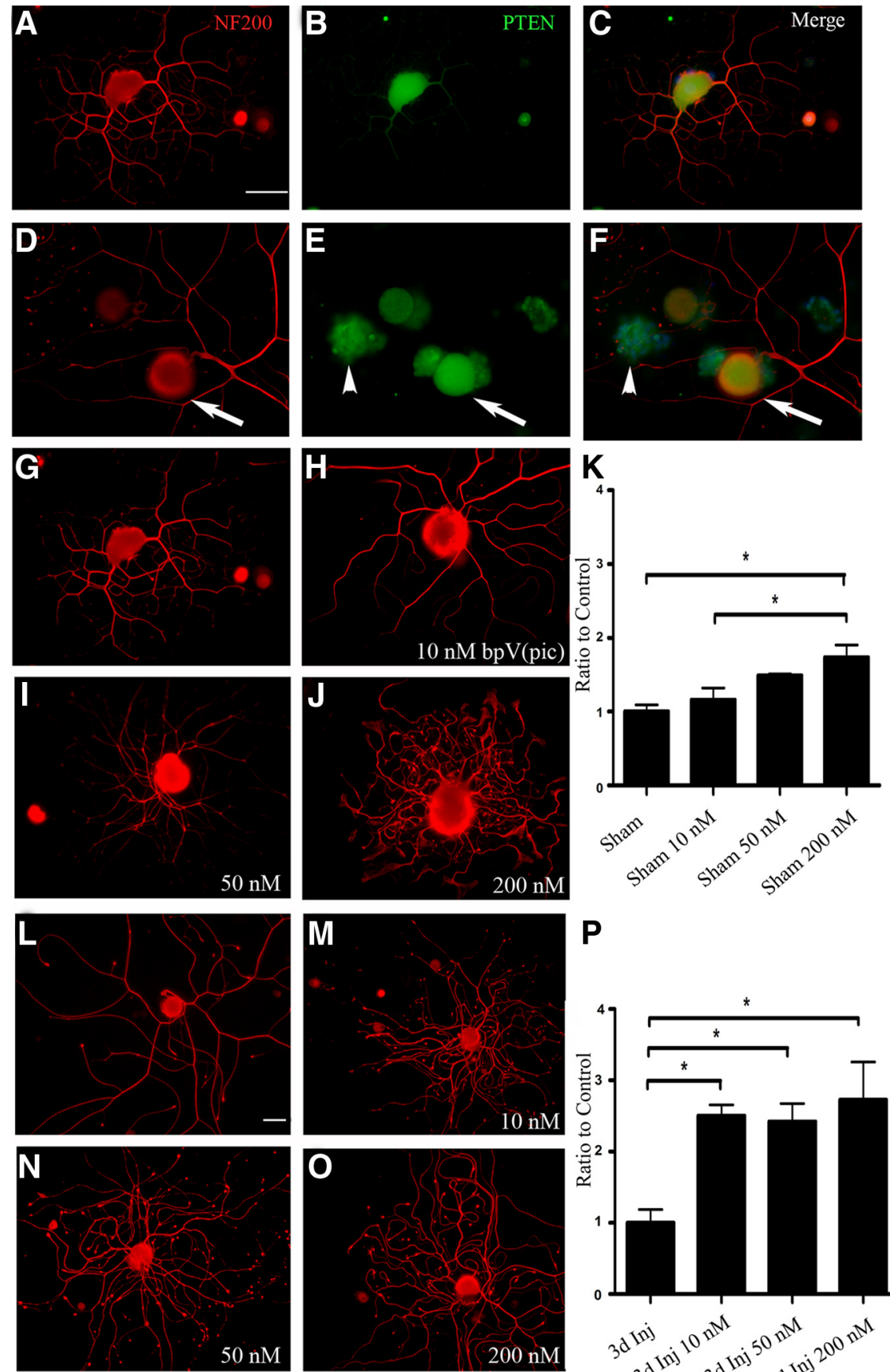

$P$
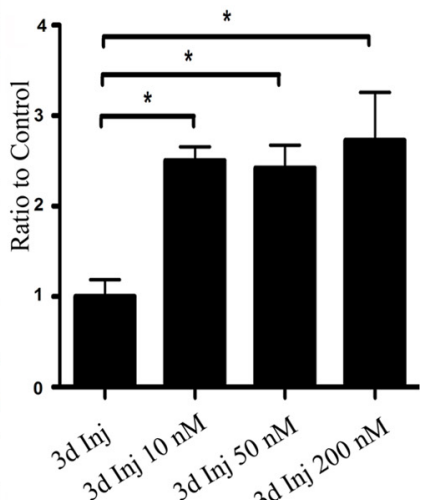

Figure 5. Inhibition of PTEN phosphatase activity increases neurite outgrowth in vitro in both sham-injured and preconditionally injured neurons. $\boldsymbol{A}-\boldsymbol{C}$, PTEN is expressed in cultured DRG neurons. NF200 (A) and PTEN (B) staining in sham-injured DRG neurons. D-F, NF200 (D) and PTEN (E) staining in injured DRG neurons. Merged images $(\boldsymbol{C}, \boldsymbol{F})$ show DAPI staining (blue). Arrow shows neuron expression, arrowhead shows glial expression. G-J, NF200 staining of sham DRG neurons with $0 \mathrm{~nm}(\boldsymbol{G}), 10 \mathrm{~nm}(\boldsymbol{H}), 50 \mathrm{~nm}(\boldsymbol{I})$, and $200 \mathrm{~nm}(\boldsymbol{J})$ of the PTEN phosphatase inhibitor, bpV(pic). $\boldsymbol{K}$, Neurite outgrowth summary from sham neurons. Outgrowth was normalized to control cultures for each sample. $\mathbf{L}-\mathbf{0}, \mathbf{N F 2 0 0}$ staining of injured cultured DRG neurons with $0 \mathrm{~nm}(\boldsymbol{L}), 10 \mathrm{~nm}(\boldsymbol{M}), 50 \mathrm{~nm}(\boldsymbol{N})$, and $200 \mathrm{~nm}(\boldsymbol{O})$ of bpV(pic). $\boldsymbol{P}$, Neurite outgrowth summary from injured neurons. Outgrowth was normalized to control cultures for each sample. Asterisks indicate significant differences (one-way ANOVA with Tukey post hoc analysis, $p<0.05, n=3$ separate cultures). Scale bars are $50 \mu \mathrm{m}$. Error bars represent SEM.

arrow shows neuron expression, arrowhead shows glial expression). To address neuron-specific plasticity, we examined neurite outgrowth in adult rat sensory neurons during graded exposure to a specific pharmacological PTEN phosphatase inhibitor (Schmid et al., 2004), bpV(pic) (10-200 nM), over $18 \mathrm{~h}$ of growth. The growth medium contained small concentrations of the growth factors insulin and NGF. There was an increase in neurite outgrowth when PTEN was inhibited (200 nM) (average neurite outgrowth from $2131.3 \mu \mathrm{m}$ to 3395.5 $\mu \mathrm{m}$ ) (Fig. 5G-K; supplemental Fig. 1, available at www.jneurosci.org as supplemental material).

Next we examined sensory neurons that had undergone a preconditioning injury, generated by sciatic nerve axotomy $3 \mathrm{~d}$ before harvesting. Preconditioning dramatically ramps up regenerative neurite outgrowth, a mechanism that involves, among others, heightened intracellular cAMP and increased expression of many regenerationassociated genes, including $\beta$ III tubulin, GAP43/B50, and others (McQuarrie and Grafstein, 1973; McQuarrie et al., 1977; Forman et al., 1980; Oblinger and Lasek, 1984; Schreyer and Skene, 1991). Our prediction was that preconditioning would suppress PTEN activity, so that little added benefit would accrue from its inhibition. However, preconditioned injured sensory neurons were yet more sensitive to $\mathrm{bpV}$ (pic) at the lowest doses applied (10 nM) and exhibited further robust rises in neurite plasticity beyond those expected of preconditioning alone (average neurite outgrowth from 5431 to $13,296 \mu \mathrm{m}$ ) (Fig. $5 L-P$ ). There was a reciprocal rise in phosphorylated Akt expression after PTEN inhibition, with no appreciable change in total Akt levels (Fig. 6; supplemental Fig. 2, available at www. jneurosci.org as supplemental material). The overall findings suggested that PTEN expressed in adult neurons actively suppressed intrinsic outgrowth properties, and inhibiting it promotes outgrowth beyond that of the preconditional nerve injury, which until now has been shown to be associated with the best outcome for axon growth in vitro.

\section{PTEN inhibition-induced neurite} outgrowth is independent of mTOR Park et al. (2008) have recently suggested that PTEN inhibition facilitates growth of central neurons through the mTOR pathway. In adult sensory neurons, we repeated our analysis of PTEN pharmacological blockade with concurrent inhibition of the mTOR pathway using rapamycin $(50 \mathrm{nM})$. Accelerated neurite outgrowth from PTEN inhibition was unaltered by rapamycin despite its active suppression of pS6 expression, a downstream effector of mTOR (Fig. 7; supplemental Fig. 3, available at www.jneurosci.org as supplemental material). Overall, the findings indicated that in peripheral nervous system regeneration, $\mathrm{mTOR} / \mathrm{pS} 6$ did not mediate accelerated neurite outgrowth following PTEN inhibition. 


\section{Inhibition of PTEN mRNA translation in vitro increases neurite outgrowth}

We next asked whether adult sensory neurons might have translation of PTEN during neurite outgrowth, which may suppress overall growth. We transfected neurons with a labeled siRNA to PTEN and examined its expression and impact on neurite outgrowth. Fluorescently tagged siRNA localized to the nucleus, cytoplasm, and neurites of DRG neurons (Fig. $8 \mathrm{~A}$ ). It also appeared to colocalize with GW bodies that label sites of mRNA degradation (data not shown, Jakymiw et al., 2005). The siRNA application protocol was associated with a transfection rate of $\sim 70 \%$ of neurons (data not shown), and there was a $50 \%$ knockdown in PTEN mRNA expression (Fig. $8 B)(p<0.001, n=8)$. PTEN siRNA dramatically increased neurite outgrowth of both sham-injured and preconditioned injured sensory neurons compared to control and scrambled siRNA application (Fig. 8; supplemental Fig. 4, available at www.jneurosci.org as supplemental material). This experiment was verified with a second PTEN siRNA targeting a separate region; PTEN expression was decreased and neurite outgrowth was significantly increased (supplemental Fig. 5, available at www.jneurosci.org as supplemental material). These findings support the pharmacological studies indicating that PTEN is normally expressed and actively translated in sensory neurons, and it functions to hinder intrinsic regenerative outgrowth of both sham-injured and preconditioned injured sensory neurons.

\section{In vivo inhibition of PTEN increases axonal outgrowth}

Given the findings that PTEN inhibition promotes neurite outgrowth, we explored its impact in an in vivo model. Homozygous deletion of PTEN is embryonic lethal, indicating that a local knockdown, rather than knock-out, of PTEN protein or mRNAs was needed to test our paradigm. We chose a complete nerve trunk transection injury, which is a severe injury with substantial limitations in outgrowth. This injury allowed us to test PTEN inhibition within a complex microenvironment, as PTEN was found in both axons and accompanying SCs. PTEN inhibition in partnering cells may boost their migration and growth leading to an overall enhancement of regeneration. We exploited a strategy that allowed serial administration of fresh reagents within the in vivo nerve regenerative milieu. A transected sciatic nerve with a 3-5 mm gap between the proximal and distal stumps was bridged by a silicone regeneration chamber (conduit) connected by a $\mathrm{T}$ junction to a subcutaneous access port as previously described (McDonald and Zochodne, 2003). In prior work, inhibition

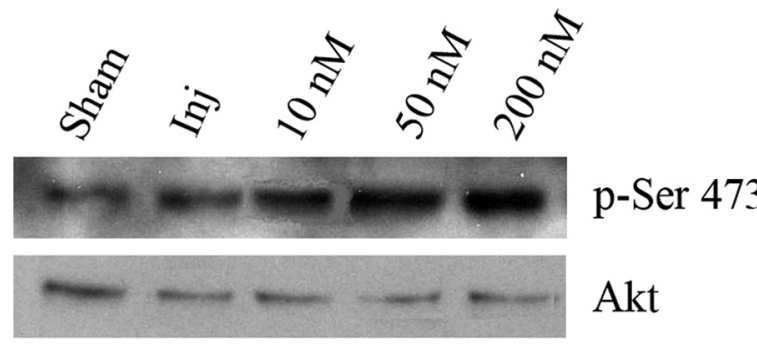

Figure 6. Increased doses of $\mathrm{bpV}$ (pic) results in a graded rise in expression of pAkt in vitro. Western blot of pAkt (Ser 473,60 kDa) and Akt in cultured 3 dinjured and sham-injured neurons with increasing doses $(10,50,200 \mathrm{~nm})$ of PTEN inhibitor.
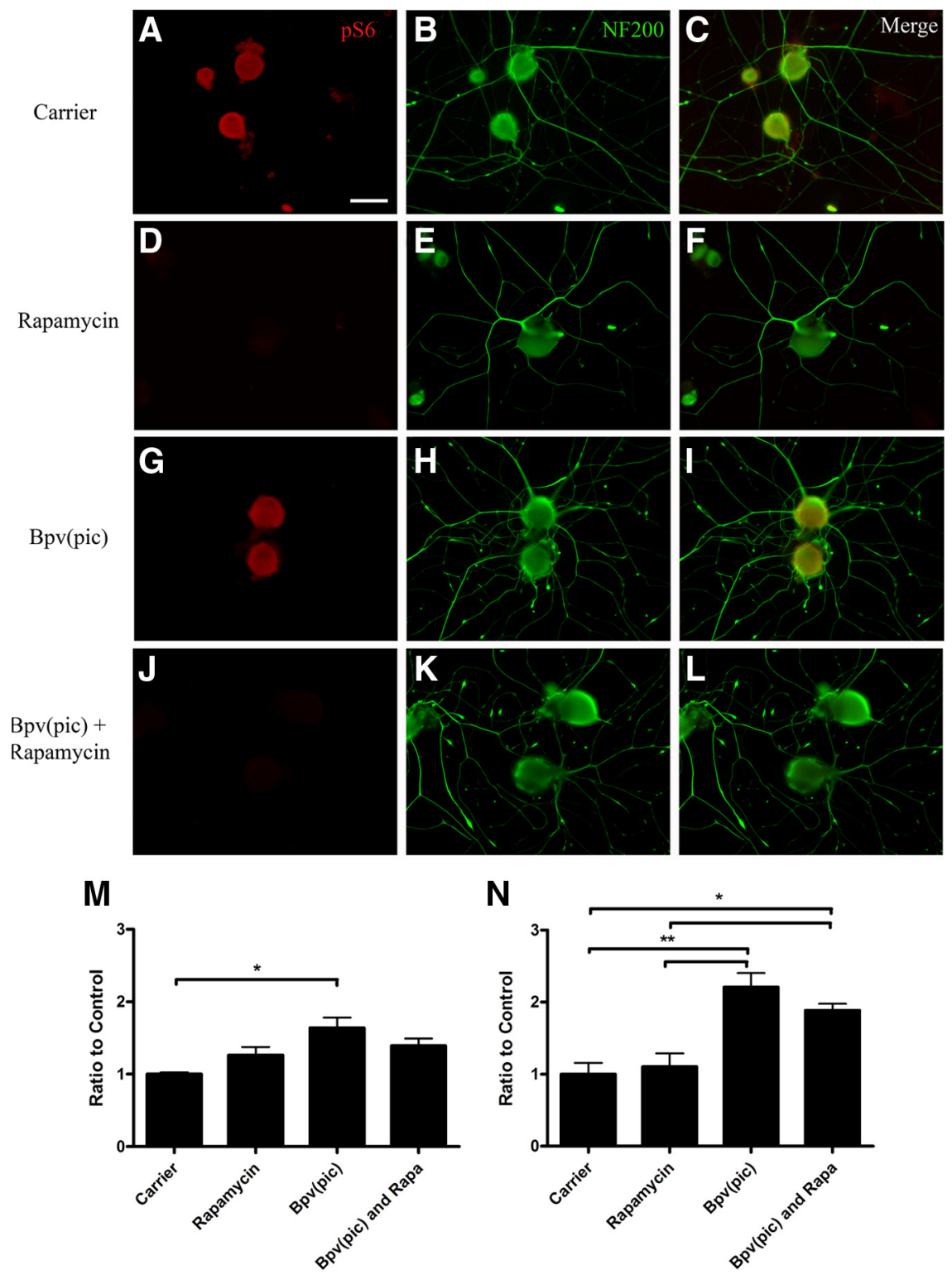

Figure 7. Application of rapamycin $(50 \mathrm{~nm})$ alone or in combination with $\mathrm{bpV}(\mathrm{pic})$ does not alter neurite outgrowth in shaminjured or preconditionally injured cultured neurons. mTOR activity is assessed with pS6K staining. $\boldsymbol{A}-\boldsymbol{C}$, pS6K (A) and NF200 (B) staining and merge $(\boldsymbol{C})$ in injured neurons exposed to carrier alone. $\boldsymbol{D}-\boldsymbol{F}$, pS6K $(\boldsymbol{D})$ and NF200 $(\boldsymbol{E})$ staining and merge $(\boldsymbol{F})$ in injured neurons exposed to rapamycin alone. $\mathbf{G}-\boldsymbol{I}, \mathrm{pS} 6 \mathrm{~K}(\boldsymbol{G})$ and $\mathrm{NF200}(\boldsymbol{H})$ staining and merge $(\boldsymbol{I})$ in injured neurons with bpV(pic) alone. $\boldsymbol{J}-\boldsymbol{L}, \operatorname{pS6K}(\boldsymbol{J})$ and NF200 $(\boldsymbol{K})$ staining and merge $(\boldsymbol{L})$ in injured neurons with bpV(pic) and rapamycin. $\boldsymbol{M}, \boldsymbol{N}$, Summary plots of neurite outgrowth in sham $(\boldsymbol{M})$ and injured $(\boldsymbol{N})$ cultures. Outgrowth was normalized to control cultures for each sample. Asterisks indicate significant differences (one-way ANOVA with Tukey post hoc analysis, ${ }^{*} p<0.05,{ }^{* *} p<0.01, n=3$ ). Scale bars are 50 $\mu \mathrm{m}$. Error bars represent SEM. 
A

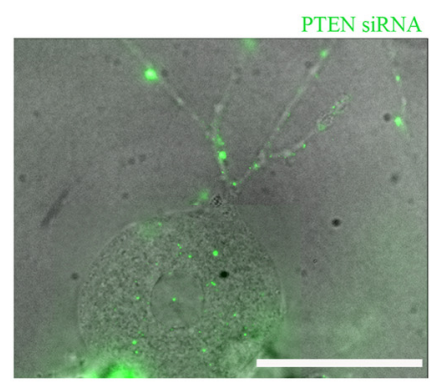

B

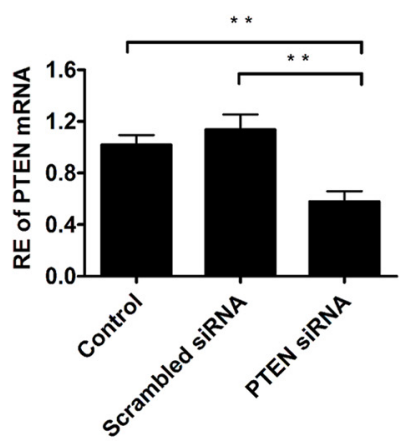

Control
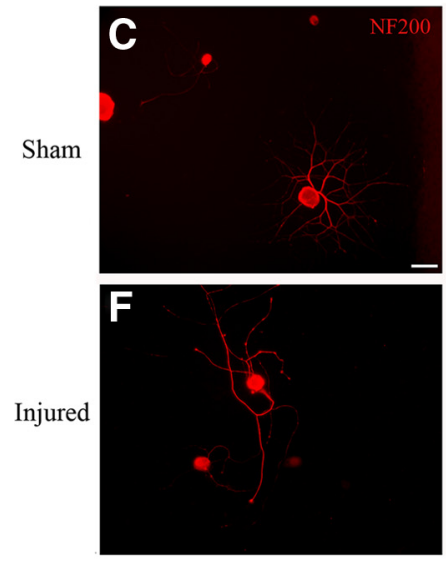

I

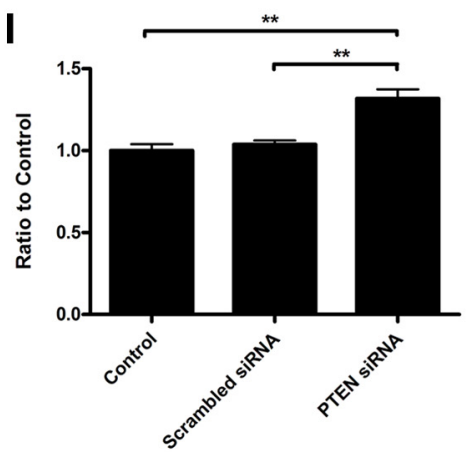

Scrambled siRNA
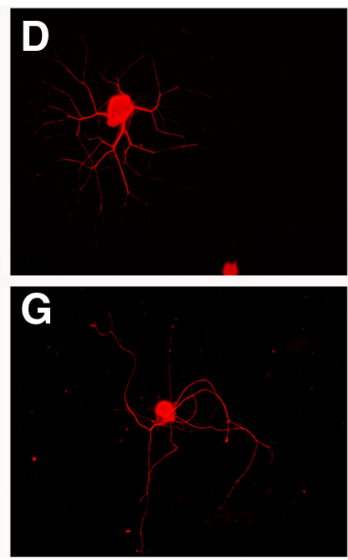

\section{$\mathbf{J}$}

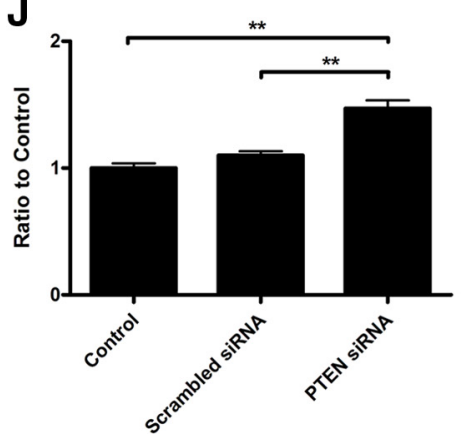

Figure 8. PTEN siRNA knocks down PTEN mRNA and increases neurite outgrowth in vitro. $A, P T E N$ siRNA localizes to DRG sensory neuron nucleus, soma, and neurites. $\boldsymbol{B}$, qRT-PCR from cultured neurons with PTEN siRNA $(p<0.001, n=8)$. $C-\boldsymbol{E}$, NF200 staining of sham-injured neurons without siRNA ( $($ ) and with scrambled siRNA (D) and PTEN siRNA (E). $\boldsymbol{F}-\boldsymbol{H}$, NF200 staining of injured neurons without siRNA $(\boldsymbol{F})$ and with scrambled siRNA $(\boldsymbol{G})$ and PTEN siRNA $(\boldsymbol{H}) . \boldsymbol{I}, \boldsymbol{J}$, Summary plots of neurite outgrowth for sham-injured $(\boldsymbol{I})$ and injured $(\boldsymbol{J})$ cultured neurons. Asterisks indicate significant differences (one-way ANOVA with Tukey post hoc analysis, $p<0.0001, n=4$ separate cultures). Scale bar is $50 \mu \mathrm{m}$. Error bars represent SEM.

of SC proliferation, RHOA inactivation, and NGF administration have each altered outgrowth using this assay (Kemp et al., 2007; Cheng et al., 2008). In vivo pharmacological inhibition of PTEN using bpV(pic) resulted in an increase in the number and length of outgrowing axons from the proximal stump of the transected nerve into the regenerative bridge (Fig. $9 A-C$ ). Interestingly, there was an increase in axonal profiles with $\mathrm{bpV}$ (pic) (Fig. 9C), which is suggestive of heightened sprouting within the proximal stump. To determine whether PTEN inhibition by siRNA exposure also increased axon and SC growth, PTEN siRNA was injected into the regeneration chamber for the $7 \mathrm{~d}$ experiment. PTEN siRNA, but not scrambled sequence siRNA (control), was associated with a $46 \%$ knockdown of local PTEN mRNA (data not shown). As in the pharmacological studies, there was an increase in the number and length of outgrowing axons and SCs from the prox- imal stump of the transected nerve into the regenerative bridge (Fig. 9D-F). Overall, these studies provided evidence that the benefits of PTEN inhibition significantly improved regeneration in vivo.

\section{Discussion}

Peripheral nerves are essential connections between the brain and body. Peripheral nerve injury and neuropathies are severely debilitating, often resulting in paralysis or pain. Current therapies are largely ineffective in restoring functional recovery. Therefore, novel strategies for increased regeneration are needed. Our results identify a new and potent strategy for promoting the regrowth of injured peripheral nerve axons. We provide evidence that PTEN has ongoing expression in the peripheral nervous system, prominent in neurons but also present in SCs, key partners in the regenerative process. PTEN is therefore present at the right time and place to be involved in nerve regeneration. Our study highlights the following: (1) PTEN is present within peripheral neurons at the injury site, where it is poised to dampen regenerative activity; (2) inhibition of PTEN increases neurite outgrowth in vitro, with a particularly striking augmentation of the already heightened growth state of a preconditioned lesion; (3) contrary to CNS regeneration, mTOR is not the mediator of this enhancement; (4) in vivo knockdown of PTEN increases axonal outgrowth following a complete sciatic nerve transection injury, indicating its active role in a complex regenerative microenvironment.

While we show PTEN mRNA and protein expression in all peripheral neurons, PTEN expression was particularly intense in small neurons coexpressing the IB4 lectin. IB4 neurons are nociceptive and innervate the epidermis (Hunt and Mantyh, 2001; Lu et al., 2001). Interestingly, IB4 neurons have been shown to have a reduced ability to regenerate in vitro, and following nerve injury in vivo, the projections from IB4 neurons retreat, resulting in hyperalgesia (Doubell et al., 1997; Leclere et al., 2007). This reduced growth capacity could be a feature linked to the high PTEN expression we identified within them, suggesting that PTEN inhibition may have a prominent role in the recovery of nociception after nerve injury. PTEN was also expressed within the intact sciatic nerve in both axons and SCs directly at the site of early regenerative activity following injury. The presence of PTEN in the proximal nerve stump following transection suggests it may play a role in suppression of regeneration. In support of this concept, we observed concurrent reductions in pAkt and pGSK $3 \beta$ expression within the injury milieu where PTEN was also expressed. Decreased phosphorylation of these molecules leads to growth cone collapse and a less supportive microenvironment at the nerve trunk injury site (Eickholt et al., 2002; Jones 
et al., 2003; Chadborn et al., 2006). Our data have confirmed an active role of PTEN through pharmacological and knockdown approaches in adult peripheral neurons. In vitro inhibition of PTEN showed a direct activation of the PI3K pathway through increased phosphorylation of Akt. Interestingly, inhibition of PTEN demonstrated accelerated growth and neurite plasticity above that of a preconditioned lesion, which were likely mediated by rises in pAkt. Moreover, in vivo inhibition of PTEN markedly increased the growth response of regenerating axons. Thus, we show that PTEN contributes to ongoing inhibition of the PI3K pathway and that removing its inhibition promotes peripheral nerve regeneration.

We are not aware of other reported strategies that accelerate regenerative activity beyond that which follows nerve preconditioning. PTEN expression specifically within the injury milieu was not downregulated following injury. Preconditioning is associated with a dramatic ramp up of regenerative activity, and supports regrowth across inhibitory substrates (David and Aguayo, 1981; Schreyer and Skene, 1991). However, in this work, we noted that rises in axon outgrowth following PTEN pharmacological inhibition were yet more prominent in neurons that were preconditioned, arguing that its role assumes even greater importance in a preconditioning scenario. While some strategies, such as bolstering neuron cAMP levels, have replicated the benefits of preconditioning (Qiu et al., 2002), none have synergistically added to the impact of preconditioning. While not addressed in this work, because of our emphasis on the challenges of peripheral neuron outgrowth, examining the impact of PTEN inhibition across central inhibitory substrates would be of interest.

The findings in our work support an initial finding that has been reported in central nervous pathway reorganization, where PTEN inhibition, through a deletion construct paradigm, was associated with increased regrowth of retinal ganglion cells into the optic nerve (Park et al., 2008). In this work, PTEN was knocked down through intravitreal injection of adeno-associated virus expressing Cre in mice with floxed PTEN ${ }^{\mathrm{f} / \mathrm{f}}$ mice. PTEN was judged to suppress mTOR phosphorylation activation of ribosomal S6kinase, a regulator of protein translation. Importantly and in contrast, we show activation of the PI3K pathway through inhibition of PTEN that is independent of MTOR pathway. There are, however, a number of additional mechanisms by which PTEN might impact regenerative outgrowth beyond PI3K and mTOR: inhibition of focal adhesion kinase in growth cones, activation of Rac1 and CDC42 GTPases, inhibition of MAP kinase signaling,

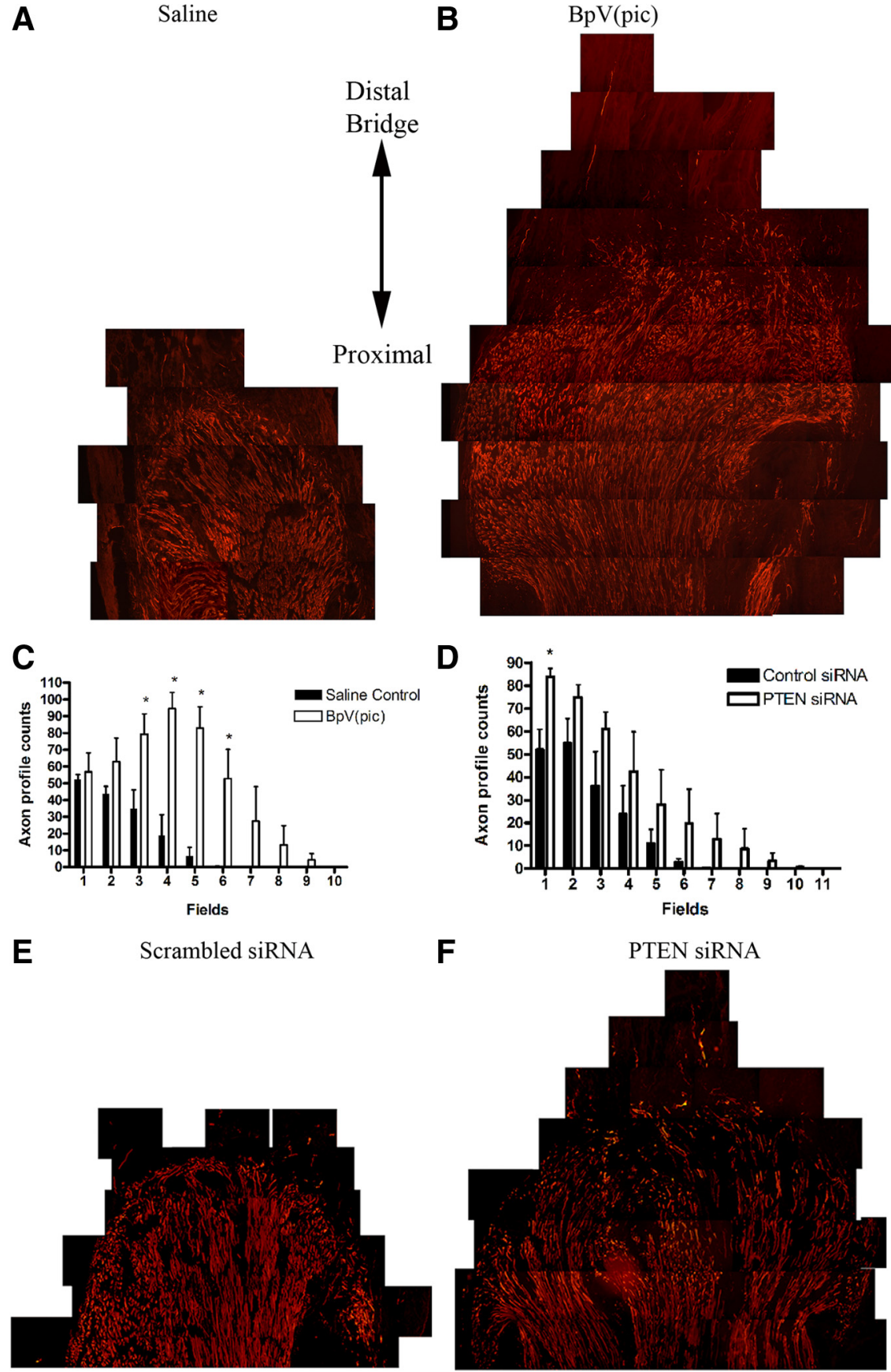

Figure 9. In vivo inhibition of PTEN at both the phosphatase and $m$ RNA levels increases the number and length of regenerating profiles from transected peripheral nerves. A, NF200 staining of a regenerative bridge treated with saline. $B$, NF200 staining of a regenerative bridge treated with $\mathrm{bpV}$ (pic) (200 nm). C, Summary of the regenerating axon profile count with bpV(pic). D, Summary of the regenerating axon profile count with PTEN siRNA. $E$, NF200 staining of a regenerative bridge treated with scrambled siRNA. $\boldsymbol{F}$, NF200 staining of a regenerative bridge treated with PTEN siRNA. Asterisks indicate significant differences $(t$ test, $p<0.05$, $n=3)$. Error bars represent SEM.

inhibition of matrix metalloproteinase-9, inhibition of receptor tyrosine kinases, and others (Papakonstanti et al., 2007) (for review, see Yin and Shen, 2008). Also, we identified expression of pPTEN within neuronal nuclei, perhaps suggestive of a role in transcriptional regulation (Rankin et al., 2009). Our approach also may also be more amenable to clinical translation to human nerve injury, since our inhibition of PTEN was temporary and local, an important consideration when inhibiting a tumor suppressor molecule.

In vivo, we confirmed that the strategy for inhibiting PTEN retains and sustains its robust impact, despite the possibility that 
expression of PTEN in other cell types, such as SCs, at the injury site could have confounded our investigation. Moreover, both pharmacological and knockdown approaches identified a large effect on early regenerative outgrowth. The findings are persuasive evidence that manipulation directly within the regenerative milieu of this critical regeneration-related signaling cascade in axons and cellular partners may provide a novel approach toward reversing regenerative failure in peripheral neurons. An important caveat is that the apparent benefits we have identified require study in long-term models of regeneration, where reconnection to distal target organs can be assessed. While it is promising, it will be important to determine whether PTEN inhibition results in accelerated functional recovery from nerve injury, work beyond the scope of these studies. Nonetheless, shutting down a key intrinsic barrier for peripheral neuron outgrowth may be synergistic with other strategies for improving the outcome of injuries and disease of peripheral neurons.

\section{References}

Ali IU, Schriml LM, Dean M (1999) Mutational spectra of PTEN/MMAC1 gene: a tumor suppressor with lipid phosphatase activity. J Natl Cancer Inst 91:1922-1932.

Chadborn NH, Ahmed AI, Holt MR, Prinjha R, Dunn GA, Jones GE, Eickholt BJ (2006) PTEN couples Sema3A signalling to growth cone collapse. J Cell Sci 119:951-957.

Chen YY, McDonald D, Cheng C, Magnowski B, Durand J, Zochodne DW (2005) Axon and Schwann cell partnership during nerve regrowth. J Neuropathol Exp Neurol 64:613-622.

Cheng C, Webber CA, Wang J, Xu Y, Martinez JA, Liu WQ, McDonald D, Guo GF, Nguyen MD, Zochodne DW (2008) Activated RHOA and peripheral axon regeneration. Exp Neurol 212:358-369.

Chin PC, Majdzadeh N, D'Mello SR (2005) Inhibition of GSK3beta is a common event in neuroprotection by different survival factors. Brain Res Mol Brain Res 137:193-201.

David S, Aguayo AJ (1981) Axonal elongation into peripheral nervous system "bridges" after central nervous system injury in adult rats. Science 214:931-933

Doubell TP, Mannion RJ, Woolf CJ (1997) Intact sciatic myelinated primary afferent terminals collaterally sprout in the adult rat dorsal horn following section of a neighbouring peripheral nerve. J Comp Neurol 380:95-104.

Eickholt BJ, Walsh FS, Doherty P (2002) An inactive pool of GSK-3 at the leading edge of growth cones is implicated in Semaphorin 3A signaling. J Cell Biol 157:211-217.

Forman DS, McQuarrie IG, Labore FW, Wood DK, Stone LS, Braddock CH, Fuchs DA (1980) Time course of the conditioning lesion effect on axonal regeneration. Brain Res 182:180-185.

Hunt SP, Mantyh PW (2001) The molecular dynamics of pain control. Nat Rev Neurosci 2:83-91.

Jain A, Brady-Kalnay SM, Bellamkonda RV (2004) Modulation of Rho GTPase activity alleviates chondroitin sulfate proteoglycan-dependent inhibition of neurite extension. J Neurosci Res 77:299-307.

Jakymiw A, Lian S, Eystathioy T, Li S, Satoh M, Hamel JC, Fritzler MJ, Chan EK (2005) Disruption of GW bodies impairs mammalian RNA interference. Nat Cell Biol 7:1267-1274.

Jones DM, Tucker BA, Rahimtula M, Mearow KM (2003) The synergistic effects of NGF and IGF-1 on neurite growth in adult sensory neurons: convergence on the PI 3-kinase signaling pathway. J Neurochem $86: 1116-1128$.
Kemp SW, Walsh SK, Zochodne DW, Midha R (2007) A novel method for establishing daily in vivo concentration gradients of soluble nerve growth factor (NGF). J Neurosci Methods 165:83-88.

Korhonen JM, Saïd FA, Wong AJ, Kaplan DR (1999) Gab1 mediates neurite outgrowth, DNA synthesis, and survival in PC12 cells. J Biol Chem 274:37307-37314.

Leclere PG, Norman E, Groutsi F, Coffin R, Mayer U, Pizzey J, Tonge D (2007) Impaired axonal regeneration by isolectin B4-binding dorsal root ganglion neurons in vitro. J Neurosci 27:1190-1199.

Lindsay RM (1988) Nerve growth factors (NGF, BDNF) enhance axonal regeneration but are not required for survival of adult sensory neurons. J Neurosci 8:2394-2405.

Lu J, Zhou XF, Rush RA (2001) Small primary sensory neurons innervating epidermis and viscera display differential phenotype in the adult rat. Neurosci Res 41:355-363.

Lyons WR, Woodhall B (1949) Atlas of peripheral nerve injuries. Philadelphia: Saunders.

McDonald D, Cheng C, Chen Y, Zochodne D (2006) Early events of peripheral nerve regeneration. Neuron Glia Biol 2:139-147.

McDonald DS, Zochodne DW (2003) An injectable nerve regeneration chamber for studies of unstable soluble growth factors. J Neurosci Methods 122:171-178.

McQuarrie IG, Grafstein B (1973) Axon outgrowth enhanced by a previous nerve injury. Arch Neurol 29:53-55.

McQuarrie IG, Grafstein B, Gershon MD (1977) Axonal regeneration in the rat sciatic nerve: effect of a conditioning lesion and of dbcAMP. Brain Res 132:443-453.

Niederöst B, Oertle T, Fritsche J, McKinney RA, Bandtlow CE (2002) Nogo-A and myelin-associated glycoprotein mediate neurite growth inhibition by antagonistic regulation of RhoA and Rac1. J Neurosci 22:10368-10376.

Oblinger MM, Lasek RJ (1984) A conditioning lesion of the peripheral axons of dorsal root ganglion cells accelerates regeneration of only their peripheral axons. J Neurosci 4:1736-1744

Papakonstanti EA, Ridley AJ, Vanhaesebroeck B (2007) The p110 delta isoform of PI3-kinase negatively controls RhoA and PTEN. EMBO J 26 : 3050-3061

Park KK, Liu K, Hu Y, Smith PD, Wang C, Cai B, Xu B, Connolly L, Kramvis I, Sahin M, He Z (2008) Promoting axon regeneration in the adult CNS by modulation of the PTEN/mTOR pathway. Science 322:963-966.

Qiu J, Cai D, Dai H, McAtee M, Hoffman PN, Bregman BS, Filbin MT (2002) Spinal axon regeneration induced by elevation of cyclic AMP. Neuron 34:895-903.

Rankin SL, Guy CS, Mearow KM (2008) Neurite outgrowth is enhanced by laminin-mediated down-regulation of the low affinity neurotrophin receptor, p75NTR. J Neurochem 107:799-813.

Rankin SL, Guy CS, Mearow KM (2009) PTEN downregulates p75NTR expression by decreasing DNA-binding activity of Spl. Biochem Biophys Res Commun 379:721-725.

Schmid AC, Byrne RD, Vilar R, Woscholski R (2004) Bisperoxovanadium compounds are potent PTEN inhibitors. FEBS Lett 566:35-38.

Schreyer DJ, Skene JH (1991) Fate of GAP-43 in ascending spinal axons of DRG neurons after peripheral nerve injury: delayed accumulation and correlation with regenerative potential. J Neurosci 11:3738-3751.

Soltoff SP, Rabin SL, Cantley LC, Kaplan DR (1992) Nerve growth factor promotes the activation of phosphatidylinositol 3-kinase and its association with the trk tyrosine kinase. J Biol Chem 267:17472-17477.

Witzel C, Rohde C, Brushart TM (2005) Pathway sampling by regenerating peripheral axons. J Comp Neurol 485:183-190.

Yin Y, Shen WH (2008) PTEN: a new guardian of the genome. Oncogene 27:5443-5453. 